\title{
Mental extrapolation in patterns constructed from memory
}

\author{
STEVEN PINKER \\ Massachusetts Institute of Technology, Cambridge, Massachusetts \\ PAUL A. CHOATE \\ University of California, Davis, California \\ and \\ RONALD A. FINKE \\ State University of New York, Stony Brook, New York
}

\begin{abstract}
Finke and Pinker $(1982,1983)$ showed subjects an array of dots followed by an arrow in a blank field, and asked them to determine whether the arrow pointed to any of the previously seen dots. Response times were linearly related to the distance between the arrow and the nearest dot, suggesting that subjects spontaneously used an internal scanning or extrapolation process to perform the task. We replicate and extend this finding by varying the retention interval, and by employing a paradigm in which subjects' eyes are closed and the arrows are described to them using a coordinate scheme. We also show that subjects are unable to predict the form of the data when the task simply is described to them. Results suggest that mental scanning along a straight path can be performed on images reconstructed from memory, and that it does not depend on the ongoing perception of a continuous surface, on physical eye movements, or on demand characteristics.
\end{abstract}

In recent years, the phenomenon of mental image scanning has come to occupy a central position in the empirical and theoretical study of imagery. Kosslyn (1973) and Kosslyn, Ball, and Reiser (1978) showed that image scanning can be studied with considerabie precision using chronometric methods. When subjects are asked to scan or shift attention between locations in an imagined scene, the time they require varies linearly with the Euclidean distances between the corresponding locations in the actual scene ( $r=.97$ in Kosslyn, et al., 1978). Since then, data from imagery-scanning experiments have been used in three ways. First, the phenomenon itself has played an important role in debates over the format of image representation, with Kosslyn and his collaborators arguing that scanning implicates an

This research was supported by NSF Grants BNS81-14916 and BNS82-16546 and an NIH Biomedical Research Support grant awarded to the first author, by a Faculty Research grant from the University of California to the second author, and by the MIT Cognitive Science Center under a grant from the Sloan Foundation. We thank Ned Block, James Pomerantz, Molly Potter, Roger Shepard, and an anonymous reviewer for helpful comments and suggestions, and Kathleen Murphy for assistance in the preparation of the manuscript. Requests for reprints should be addressed to Steven Pinker, Department of Psychology, E10-018, Massachusetts Institute of Technology, Cambridge, MA 02139, or to Ronald Finke, Department of Psychology, State University of New York at Stony Brook, Stony Brook, NY 11794. array-like representation for imagery (e.g., Kosslyn, 1980, 1981; Kosslyn, Pinker, Smith, \& Shwartz, 1979; Kosslyn \& Pomerantz, 1977), and Anderson (1978) and Pylyshyn (1981) arguing that propositional representations can account for the data equally well or better. Second, the scanning paradigm has been employed as a mental "tape measure" in attempts to answer questions about geometric properties represented in images. For example, subjects' response times (RTs) in such experiments have been used in attempts to determine the degree of preservation of metric information in images (Kosslyn et al., 1978), the maximum width of an image (Kosslyn, 1978), the dimensionality of images (Pinker, 1980; Pinker \& Kosslyn, 1978), the accuracy of imagined displacements of objects (Pinker \& Kosslyn, 1978 ), the accessibility of perspective properties in images (Pinker, 1980; Pinker \& Finke, 1980), the role of eye movement commands in imagery (Pinker, 1980), and the sequence of operations used in mental image generation (Kosslyn, Reiser, Farah, \& Fliegel, 1983). Third, the ability to scan images has been proposed as one of the mental tools or components of "visual routines" (see Ullman, 1982) that humans can put to use in solving certain geometric problems, such as verifying the collinearity of three objects (Spoehr \& Williams, 1978), determining whether an object lies in a particular direction with respect to some reference point (Attneave \& Pierce, 1978; Finke \& Pinker, 1982), and estimating 
distances from mental maps (Reed, Hock, \& Lockhead, 1983; Thomdyke, 1981; Thomdyke \& Hayes-Roth, 1982).

Despite the potential importance of image scanning, several critics have cast doubt upon the very existence of the phenomenon or upon its significance if it does exist. Some have argued that subjects can determine the purpose of image-scanning experiments from the experimental instructions or from nonverbal cues from the experimenter, and deliberately time their responses to make them linearly related to interobject distance in an attempt to comply with the demand characteristics of the experiment (Intons-Peterson, 1983; Mitchell \& Richman, 1980; Richman, Mitchell, \& Reznick, 1979). In addition, Pylyshyn (1981) argued that the instructions in imagery tasks are ambiguous with respect to whether the task requires subjects to move an internal processing locus across their image or to entertain the notion that an episode of physical movement of an object is taking place in real time. If subjects arrive at the latter interpretation, they would have little choice but to delay their buttonpresses by the amount of time they estimate the corresponding physical event would require. If so, they would not have to scan or even form an image in order to yield RTs linearly related to interpoint distances (alternatively, they would use imagery, but imagery itself would be nothing but the use of tacit knowledge to simulate physical events).

Some of these objections were addressed in a series of studies by Finke and Pinker $(1982,1983)$. On each trial, subjects were shown a pattern of 3-5 dots for several seconds, then a blank field, and then an arrow appearing at an unpredictable location. Their task was to determine as quickly as possible whether the arrow pointed at any of the previously seen dots. Subjects' RTs were related linearly to the distance between the arrow and the location of the nearest dot to which it pointed, and subjects reported that they retained an image of the dots and mentally scanned along an imaginary extrapolation of the arrow until they encountered either an imagined dot or a region of the field containing no dots. Furthermore, the slope of the RT-distance function, an estimate of the rate of scanning, was very close to those obtained in the earlier experiments on image scanning cited above. Since there were no instructions to form or scan images, and no mention of physical motion of any sort, it is highly unlikely that subjects' spontaneous use of what appears to be an image-scanning strategy was the product of their second-guessing the experimenter about the properties of imagery or of their interpreting the task as an exercise in the mental simulation of physical motion. Finke and Pinker (1983) also showed that the image-scanning strategy was used only when the location of the arrow was unpredictable. When subjects were shown the arrow's location a sufficient amount of time before the arrow itself appeared, they were able to compute the relative direction of each dot with respect to that location and then simply matched the arrow's orientation against this set of directions directly, taking equal amounts of time for all distances. This also shows that the extrapolation task does not, in general, inspire second-guessing of the hypothesis or mental simulation because the linear RT-distance relation can either occur or not occur, depending on a factor-duration of warning interval for arrow location-that is unrelated to the task instructions.

There are still some important problems raised by the Finke and Pinker $(1982,1983)$ studies, however, and there are still doubts that image scanning is sufficiently well understood to permit the sorts of strong conclusions cited in the first paragraph. First, in those experiments, the subjects saw the arrow no more than $2 \mathrm{sec}$ after the dot pattern had disappeared. In contrast, most of the imagery-scanning studies cited earlier involved patterns committed to memory minutes or hours before the task. This raises the worry that the scanning took place in some rapidly fading short-term visual memory representation that may be distinct from images constructed from long-term memory. Aside from iconic memory and retinal afterimages, there are several candidates for such a short-term visual memory store, such as the spatiotopic buffers that have been posited to integrate information across saccades (see Breitmeyer, 1983, McConkie \& Rayner, 1976, and Potter, 1983) or the very short-term (9 $\mathrm{sec}$ or less) visual memory documented by Phillips (1974) and discussed by Potter (1983). Thus, the Finke and Pinker (1982, 1983) studies do not demonstrate that spontaneous mental scanning is possible in the form of imagery that is addressed in the imagery debate, namely, images constructed from long-term memory, only in that resulting from an immediately prior visual stimulus.

A second problem is that, in all of the experiments, subjects saw the arrow and the blank field in front of them while they were performing the task, and may have been moving their eyes along the extrapolated line from the arrow to the location of the (now absent) dot during the scanning process. This raises the following concerns. Although Finke and Pinker (1982) argued, on the basis of comparisons of scanning rates, that the linear increase in RT with distance is not a simple consequence of the time taken by physical eye movements, it is still conceivable that some slowed-down eye movement process, or one in which small saccades along the extrapolated line are alternated with fixations of constant duration, could account for the effect without the necessity of positing an internal scanning process. In addition, Pylyshyn (1981) argued that experiments in which subjects scan across or superimpose images onto visible physical surfaces do not bear on the debate about the format of imagery or the nature of image scanning. Pylyshyn proposed that, in such cases, the geometry of a veridically perceived surface forces the subject, when imagining movement along a path from one location to another, to imagine the movement traversing all locations in between. We feel Pylyshyn's argument is un- 
sound, since one can posit models of veridical perceptual representations in which intermediate locations need never be processed (e.g., if numerical coordinates were manipulated algebraically). Thus, patterns of data from such experiments are consequences of the nature of perceptual representations and processes and not of the geometry of the input. Nonetheless, the case would be stronger if one could show that spontaneous image scanning also occurs when subjects' eyes are closed and their representations are constructed entirely from memory.

Third, there was a complication in the results of Finke and Pinker (1983): Subjects made far more errors for the shortest arrow-dot distance $(3 \mathrm{~cm})$ than for the other distances. This did not betray an across-the-board speed-accuracy tradeoff, since errors were constant across the remaining range of arrow-dot distances $(4.5-12 \mathrm{~cm})$ and RTs increased linearly over that range. Finke and Pinker (1983) explained this anomaly as follows. Dot positions are not remembered with perfect accuracy, but there is a region of uncertainty surrounding each position. "Hits" and "misses" were defined in the experiments in terms of the angle between the arrow and a line joining the dot to the tail of the arrow, regardless of distance, such that in "miss" trials the arrow missed a dot by $40 \mathrm{deg}$ or more. Subjects thus should adopt a tapering in region surrounding the extrapolated line as a criterion for responding "hit" or "miss." Therefore, a constant degree of positional inaccuracy will lead to many errors for distances below a certain value, but no errors above that value (this is illustrated in Figure 1, top panel). ${ }^{1}$ Although this explanation of the anomalous data appears plausible, Finke and Pinker (1983) did not adduce independent evidence for it, and so it remains mostly conjectural.

The experiments reported in this paper are intended to address these problems. To address the problem of the short retention interval of the visual pattern, we replicated the Finke and Pinker $(1982,1983)$ experiments but varied the retention interval parametrically (in Experiment 1), and ran a related experiment in which the dot pattern was presented only at the beginning of a block of trials (Experiment 3 ). To address the problem of the visibility of the field containing the arrow and (previously) the dots, we designed Experiment 3 so that subjects could perform the entire task with their eyes closed. To address the problem of the anomalously elevated error rate for the shortest distance, we introduced two manipulations that would be expected to enhance the effect if Finke and Pinker's (1983) explanation is correct: shorter arrow-dot distances (in Experiment 1) and longer retention intervals (in Experiments 1 and 3). Finally, in Experiment 2, we exploited the anomalous data pattern itself and the effects of varying the retention interval to show that the results were unlikely to be an artifact of demand characteristics.

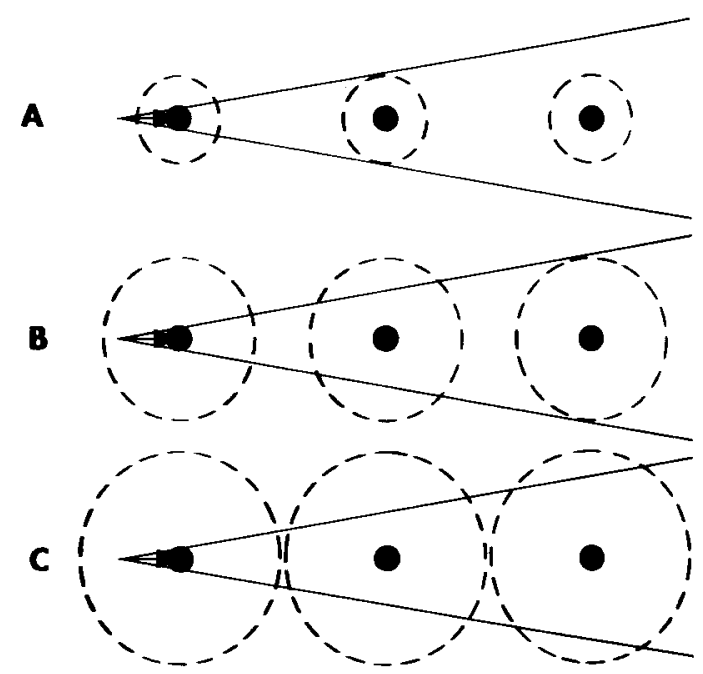

Figure 1. Illustration of proposed explanation for elevated error rates and RTs for short arrow-dot distances in mental extrapolation experiments. Arrow sectors define zones within which a remembered or imagined dot counts as a "yes" response. Dashed circular regions surrounding each dot denote the range of error in the remembered dot locations. The probability of making an error in judging whether an arrow points to a dot corresponds to the proportion of the area of the memory region that falls outside the scanning sector. Dots are shown representing distances of $0,1.5$, and $3 \mathrm{~cm}$, for short (A), medium (B), and long $(C)$ retention intervals, respectively, paralleling the conditions of Experiment 1. Higher error rates are predicted for short distances and longer retention intervals.

\section{EXPERIMENT 1}

This experiment was a replication and extension of Finke and Pinker $(1982,1983)$, such that we varied the retention interval from 0 to $10 \mathrm{sec}$ and such that the range of distances was extended below Finke and Pinker's (1983) $3-\mathrm{cm}$ minimum value. We hypothesized that, if the scanning phenomenon was not confined to very short-term visual memory, then RT would increase linearly with arrow-dot distance even at the $10-\mathrm{sec}$ retention interval, which would exceed the estimated duration of the very short-term visual buffers, other than imagery per se, that had been proposed previously (see Potter, 1983, for a review). Furthermore, the experiment allowed us to estimate the effects of retention interval on image construction and maintenance time (by examining the intercept of the function) and on the scanning rate (by examining its slope). Finally, if Finke and Pinker's (1983) elevated error rate for the shortest arrow-dot distance was a consequence of inaccuracy in remembered dot location, then any manipulation that served to increase that uncertainty (e.g., increasing the retention interval) should enhance the effect (see Figure 1). This enhancement could take the form of an increased error rate at the short distances, or of error rates being elevated over a greater range of distances at the low end of the distance scale. Figure 1 
also shows that decreasing the distance between dot and arrow should also enhance the effect, another prediction of this experiment.

\section{Method}

Subjects. Forty-five undergraduate students at the University of California at Davis served as subjects in exchange for credit in an introductory psychology course.

Procedure. The general procedure was similar to that used in Experiment 1 of Finke and Pinker (1983). The subjects were tested individually and were informed only that the experiment would be one on visual memory. A Gerbrands four-channel tachistoscope was used to display all experimental materials. At the beginning of each trial, the subjects were shown a pattern consisting of three, four, or five black dots, $6.35 \mathrm{~mm}$ in diameter, on a white background; these patterns were identical to those in Finke and Pinker (1983). A pattern was displayed for $4 \mathrm{sec}$, and the subjects were instructed to remember the location of each of the dots. Later in the trial, a solid black arrow, $1 \mathrm{~cm}$ in length, was presented on a white background, and the subjects were instructed to indicate, by pressing the appropriate button on a response box, whether the arrow was pointing to the location of any of the dots that they had just observed. For 15 of the subjects, the arrows were presented immediately after the offset of the pattern; for another 15, an empty white field was presented for $2 \mathrm{sec}$ following the offset of the pattern; and for the remaining 15 subjects, the arrow appeared $10 \mathrm{sec}$ after the offset of the pattern. In the 10-sec delay condition, the tachistoscopic field was totally dark for the first $8 \mathrm{sec}$, followed by a $2-\mathrm{sec}$ warning period during which the white blank field was presented. All of the fields were $14 \times 14 \mathrm{deg}$ in size, were viewed at a distance of $75 \mathrm{~cm}$, and were matched for average luminance.

The experiment began with an explanation of the task, followed by a display in which an arrow pointed at one of the dots in a configuration similar to those used in the experiment. The subjects were told to respond as quickly and as accurately as they could as soon as the arrows were presented. We explained that the arrows would point either directly at the center of a dot or would completely miss all of the dots in the pattern by at least $40 \mathrm{deg}$. The subjects were then given an opportunity to practice pressing the two response buttons and to respond to arrows in a series of practice trials.

The practice trials consisted of 14 presentations of a four-dot practice pattern, followed by an arrow at the appropriate delay time for each group. The arrow-to-dot distance ranged from 0 to $12 \mathrm{~cm}$ at $1.5-\mathrm{cm}$ intervals, as measured from the tip of the arrow to the center of the dot. One arrow for each of these nine distances was then presented, together with five arrows that completely missed the dots. These arrows appeared in random order, with the single constraint that there could be no more than three consecutive "yes" or two consecutive "no" trials. Error feedback was provided after each response.

The experimental trials, consisting of three sets of 28 trials each for the three-, four-, and five-dot patterns, were then conducted. Before a set of trials began, the dot pattern was inspected by the subjects for $30 \mathrm{sec}$; the patterns and arrows were then presented as in the practice session. Each set of trials contained 2 arrows for each of the nine distances, and 10 arrows that did not point to any of the dots. The arrows were evenly distributed throughout the viewing field, and no 2 arrows were ever presented from the same location. The order of the arrows was randomized as in the practice session, and the order of dot patterns was randomized across subjects within each group.

\section{Results}

Reaction times. The mean RTs to respond affirmatively and correctly that an arrow was pointing to a previously seen dot are shown in Figure 2. It can be

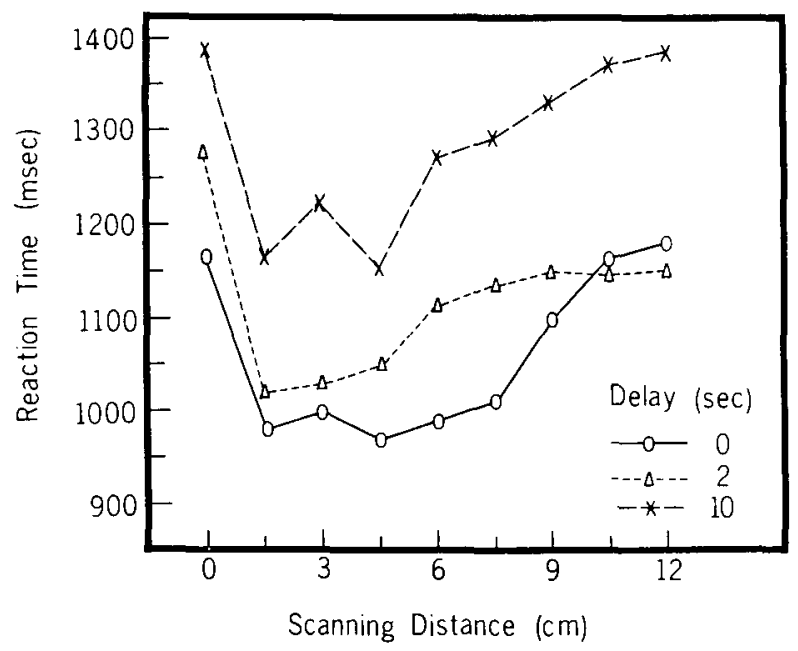

Figure 2. Mean RTs to verify correctly that an arrow pointed at a previously presented $\operatorname{dot}(\mathrm{N}=45)$.

seen that the functions relating $\mathrm{RT}$ to distance are nonmonotonic, displaying an increase in RT with in creasing distance except at the $0-\mathrm{cm}$ distance, at which the RTs are anomalously high.

An analysis of variance reveals that $\mathrm{RTs}$ varied signifi. cantly with arrow-dot distance $[F(8,336)=13.44$ $\mathrm{p}<.001]$. Trend analyses for the full distance range of $0.12 \mathrm{~cm}$ revealed highly significant linear and quadratic trends $[F(1,336)=15.01, p<.001$, and $F(1,336)=$ $48.99, \mathrm{p}<.001$, respectively]. The departure from linearity was due entirely to the elevated $0-\mathrm{cm}$ RT with this single point excluded, the linear trend was highly significant $[F(1,294)=93.80, p<.001]$, the quadratic trend was not significant $[F(1,294)=1.17$ $\mathrm{p}>.25]$, and there was no appreciable residual variatior $(F<1)$. Figure 3 displays the regression line for $R T$ : within the $1.5-12-\mathrm{cm}$ range of distances, averaged acros: the three levels of delay. It is clear that the aggregat

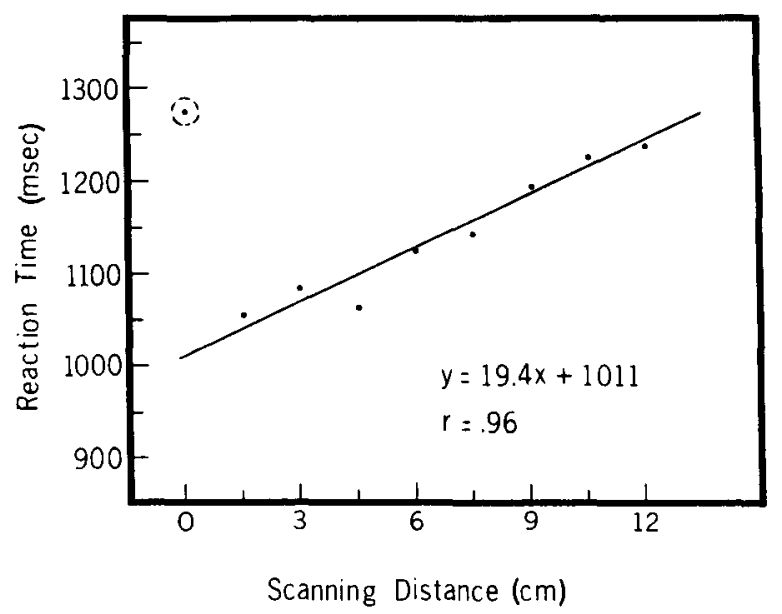

Figure 3. Linear regression for average RTs in Experiment 1 excluding the $0-\mathrm{cm}$ distance $(\mathrm{N}=45)$. 
data are highly linear $(\mathrm{r}=.96)$. The slope of this function, $19.4 \mathrm{msec} / \mathrm{cm}$, is quite close to the $21.8-\mathrm{msec} / \mathrm{cm}$ slope obtained in Experiment 1 of Finke and Pinker (1983), which was obtained using distances ranging from 3 to $12 \mathrm{~cm}$.

The effect of delay of arrow presentation did not reach significance, although it was suggestive of an overall increase in image formation on preparation time $[\mathrm{F}(2,42)=2.33, \mathrm{p}<.12]$. There was also no overall interaction between distance and delay $(F<1)$, indicating that the shape of the RT functions did not vary significantly across different arrow-presentation intervals. Note that the delay factor itself was nonsignificant despite the differences between curves apparent in Figure 2 , probably because delay, unlike distance, was a between-subjects manipulation. This implies that the nonsignificant interaction between delay and distance must be interpreted cautiously. The mean RTs for correct rejections were $1,029,999$, and $1,205 \mathrm{msec}$ for the $0-, 2-$, and $10-\mathrm{sec}$ delay conditions, respectively, and in this case the effect of delay on RT was statistically significant $[F(2,42)=3.77, p<.05]$.

Error rates. Figure 4 displays the total number of "miss" errors made for each distance in the three delay conditions. As expected, the greatest number of errors was made for the $0-\mathrm{cm}$ distance-an average rate of $37 \%$. As Figure 4 also shows, the error functions were essentially flat for all delay conditions within the $3-12-\mathrm{cm}$ range, with an average error rate of $8 \%$, similar to that obtained by Finke and Pinker $(1982,1983)$ for the identical range. Of greater interest, the error rates for the $1.5-\mathrm{cm}$ distance show that, for certain judgments, task difficulty increases with time. The rates for the $10-, 2-$, and $0-\sec$ delay conditions were, respectively, $30 \%, 21 \%$, and $8 \%$. An analysis of variance performed on the error rates for the three shortest distances (those in

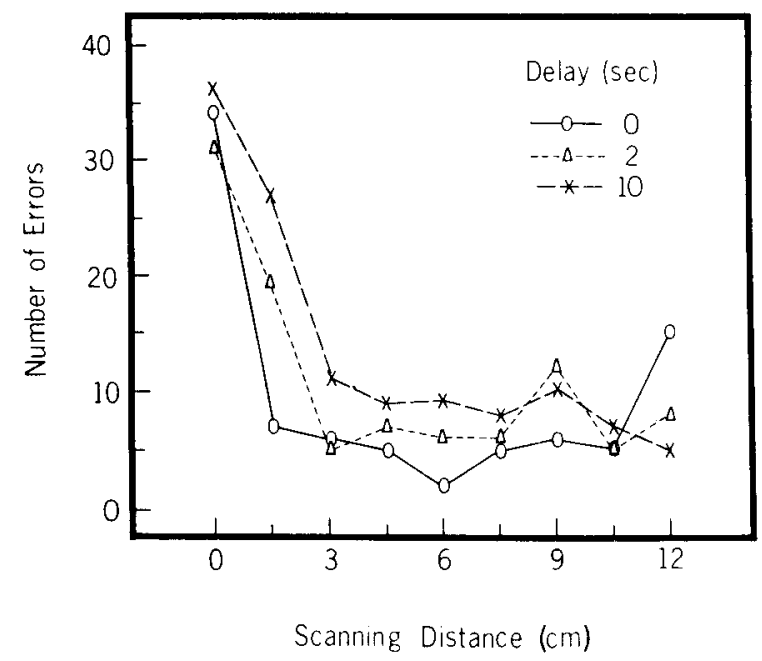

Figure 4. Mean percentage of errors in attempting to verify that an arrow pointed at a previously presented $\operatorname{dot}(\mathrm{N}=45)$. which the Finke \& Pinker, 1983, study would lead one a priori to expect elevated error rates) confirms that these differences are significant. Both the distance and the delay factors were significant $[\mathrm{F}(2,84)=46.70$, $\mathrm{p}<.001 ; \mathrm{F}(2,42)=4.28, \mathrm{p}<.02]$, and their interaction was marginally significant $[F(4,84)=2.23, p<.08]$. The rates of false alarms were $9 \%, 16 \%$, and $13 \%$ for the $0-, 2-$, and 10 -sec delay conditions, respectively $[\mathrm{F}(2,42)$ $=2.80, \mathrm{p}<.10]$.

\section{Discussion}

The results of this experiment replicate the earlier findings reported by Finke and Pinker (1982, 1983), showing that, in the present task, people spontaneously form and scan mental images even when the instructions make no mention of images, scanning, or motion. In addition, the results extend these findings to include arrow-presentation delays ranging from 0 to $10 \mathrm{sec}$. Thus, the effect is not confined to rapidly fading traces of visual input. Note, incidentally, that although we can argue that very short-term traces were not involved at the 10 -sec interval, we cannot argue that they were involved at the shorter intervals, since the same dot pattern was repeated over trials, enabling long-term visual memory to have been operative even at our shortest retention interval.

The chronometric results also reveal that, at the $0-\mathrm{cm}$ distance, there are striking departures from linearity in all the delay conditions. We attribute this sharp increase in RT to the same factor that led to a high error rate for the shortest distance in the Finke and Pinker (1983) experiments: At very short distances, even a small displacement in the remembered location of a dot can place it outside the acceptable angular range of the hit region. Most likely, this required extra effort or an additional regeneration or rechecking of the dot position by the subjects, thus increasing their RTs. By reducing the arrow-dot distance, we caused the anomaly in error rates for short distances that was observed in the Finke and Pinker (1983) study to manifest itself in the RT data (as well as in the error data) in this study, and thus have shown that the phenomenon reflects some intrinsic difficulty of this task condition and not some unusual speed-accuracy tradeoff.

This account is supported further by the error data from the present study, which suggest that the remembered locations of the dots tend to "drift" over time and that this drift has the effect predicted by the considerations displayed in Figure 1. The greatest number of errors occurred for the $0-\mathrm{cm}$ distance, with about the same number in each delay condition. For the $1.5-\mathrm{cm}$ distance, the number of errors was reduced, but much more so as the delay interval was shortened. For distances greater than $3 \mathrm{~cm}$, the error functions for all three intervals were essentially flat. This error pattern can be explained by assuming that the regions in which the imagined duts were located increased in size over 
time and that a constant judgment criterion was used for the "yes" responses. As Figure 1 shows, the greatest number of errors would be expected at the $0-\mathrm{cm}$ distance, since the scanning sector is very narrow at this point and intersects the smallest proportion of area of the memory regions. Moreover, increasing the size of these regions (by extending the delay period) would not substantially decrease this proportion, and hence the probability of making an error should not vary by large amounts. At the $1.5-\mathrm{cm}$ distance, however, increasing the size of the memory regions would increase considerably the likelihood of making an error; for the 0-sec delay condition, the region lies entirely within the scanning sector, whereas at longer delays, the scanning sector intersects progressively smaller proportions of the regions. Finally, at the $3-\mathrm{cm}$ distance and beyond, the scanning sector is wide enough to include each of the regions in their entirety, resulting in a small and relatively constant number of errors.

It should be noted that the RT functions do not correspond precisely to the error functions, because the unusually slow RTs are unique to the $0-\mathrm{cm}$ distance. This difference may reflect a tendency to sacrifice accuracy for speed except for the especially confusing case in which the arrows were presented in the immediate vicinity of the dots. This last result also helps to rule out the possibility that subjects might have been biased to respond "yes" whenever the arrow was near a previous dot location.

\section{EXPERIMENT 2}

In light of previous reports that subjects can often predict the outcome of experiments on mental image scanning (e.g., Mitchell \& Richman, 1980), we thought it would be useful to include a simple control experiment, in which subjects are encouraged to predict the results of Experiment 1. Although it seemed possible that control subjects would be able to predict the linear trends in the RT functions when the independent and dependent variables are explicitly pointed out to them, we conjectured that even then they would not be able to predict the large increase in RT at the $0-\mathrm{cm}$ distance or the particular pattern of error rates.

\section{Method}

Subjects. Forty-eight undergraduate students at the University of California at Davis served as subjects.

Procedure. The subjects were tested as a group. They were first given a full description of the judgment task and experimental materials used in Experiment 1, and were read the instructions for that experiment. A single dot display and a single arrow were drawn on the blackboard as examples. The subjects were told that their task was to try to guess the RT and number of errors that experimental subjects would make for each arrow-dot distance and each delay condition. They were then given response sheets containing spaces for all combinations of distance levels and delay conditions, and were instructed to mark their best guesses for RTs and errors in the appropriate spaces. So that the control data would fall roughly within the same range of values as the experimental data, the experimenter specified the highest and lowest RTs and error rates previously obtained, and instructed the subjects to use these values for their highest and lowest predictions, but without revaling the conditions from which these values were taken. (linke \& Pinker, 1982, pointed out that in Mitchell \& Richman's, 1980, study, which lacked such instructions, subjects' estimates of the scanning rate were off by an order of magnitude.) The predicted responses were then collected and analyzed in the same manner as the actual responses in Experiment 1.

\section{Results and Discussion}

Estimated reaction times. The mean estimated RTs, shown in Figure 5, were all highly linear as a function of distance $(\mathrm{r}=.98)$. The means increased significantly with both distance $[F(8,376)=83.99, p<.001]$ and delay $[F(2,94)=73.20, p<.001]$. In contrast to the RTs obtained in Experiment 1, the predicted times did not show a large departure from the linear trend at the 0 -cm distance. The estimates also differ from the corresponding experimental data in that RT was predicted to increase with distance more rapidly with increasing delay of arrow presentation $[F(16,752)=11.29, p<$ $.001]$, instead of increasing at roughly similar rates across delay conditions.

Since our control subjects were able to predict the linear nature of the RT functions, these findings agree with those of earlier studies, such as that of Mitchell and Richman (1980), in which subjects were told the independent and dependent variables of a typical imagery. scanning experiment and were asked to guess their relationship (note that when independent and dependent variables are not pointed out, as in real scanning experiments, subjects are unlikely to guess the purpose of the experiment; Kosslyn et al., 1979). In any case, the absence of an increase in predicted RT at the $0-\mathrm{cm}$ distance argues that, in this experimental task, subjects do not, in general, respond according to their performance expectations (see Reed et al., 1983, for another demonstration of subjects' being unable to predict chronometric patterns in image scanning). And even if

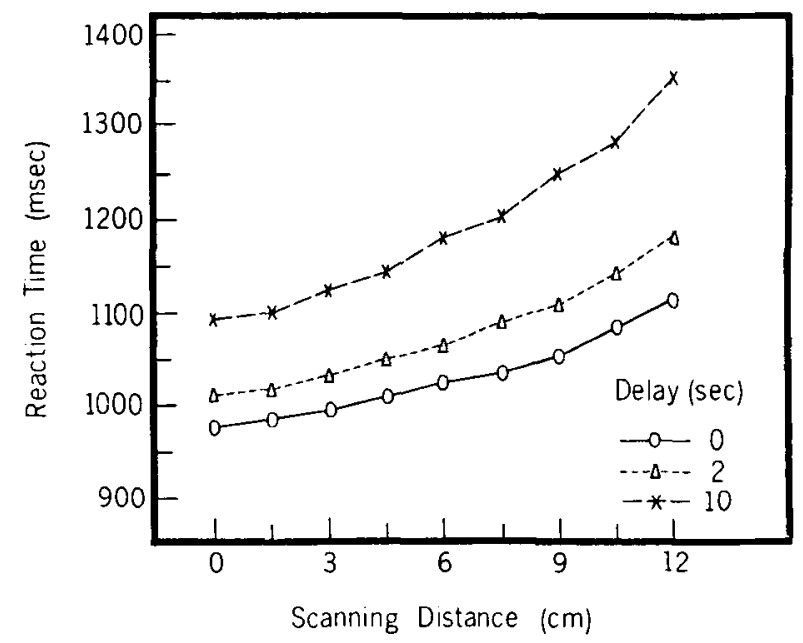

Figure 5. Mean RTs predicted by control subjects in Experiment $2(\mathrm{~N}=45)$. 


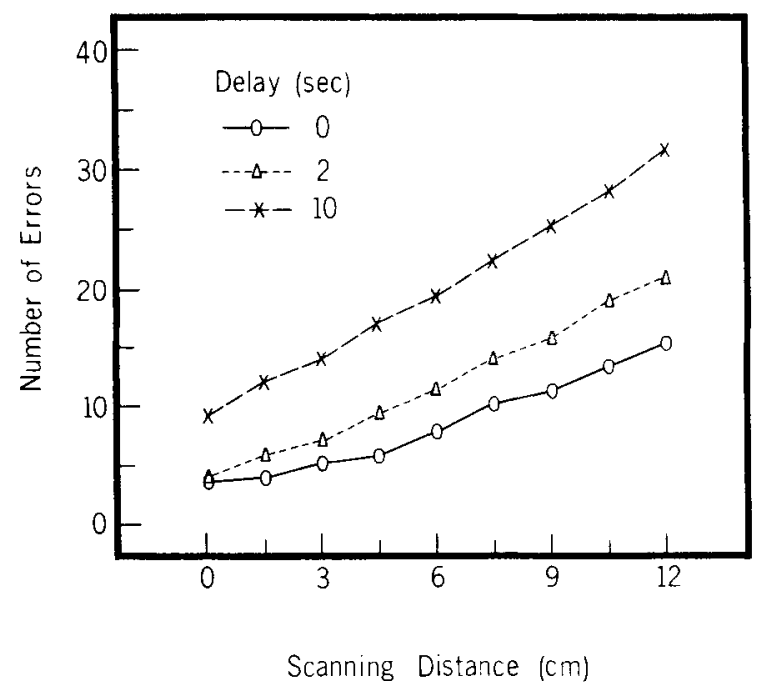

Figure 6. Mean percentage of errors predicted by control subjects in Experiment $2(\mathrm{~N}=48)$.

our experimental subjects had attempted to perform according to these expectations, they first would have had to determine the arrow-dot distance at the moment they were supposed to give their responses (since distance information was not provided in advance), which should have been least difficult to do for the $0-\mathrm{cm}$ distance.

Estimated error rates. As Figure 6 reveals, the predicted error functions increased monotonically and linearly with increasing arrow-dot distance, corresponding to the predicted RT functions. This is in sharp contrast to the actual error functions in Experiment 1, which at the low end of the distance scale decreased rapidly with increasing arrow-dot distance, and which were flat for distances greater than $3 \mathrm{~cm}$. Clearly, the pattern of error rates for this task cannot be attributed to subjects' performance expectations. ${ }^{2}$

It is possible, of course, that subjects in real experiments deliberately time their responses and make incorrect choices on a predetermined proportion of trials for distances of $3 \mathrm{~cm}$ and greater, and that only the trends observed for distances of less than $3 \mathrm{~cm}$ genuinely reflect the duration and error likelihood of mental processes. However, given the number of discrepancies between estimated and actual data patterns, the fact that the task instructions did not call for scanning or for any other process related to arrow-dot distance, the systematic patterns of error rates in the real experiments (which should not have been deliberately manipulated if subjects were simply simulating physical motion), and the absence of distance information prior to the arrow stimulus, accounts based on demand characteristics must become increasingly ad hoc and implausible. ${ }^{3}$

\section{EXPERIMENT 3}

As mentioned in the introduction, in the Finke and Pinker (1982, 1983) studies, and in Experiment 1, the arrow and the field formerly occupied by the dots were visible throughout the time in which the subjects were scanning. This could be a problem, in light of Pylyshyn's (1981) argument that linear RT-distance findings in such a case are consequences of the geometry of surfaces and the veridicality of perception, and in light of the concern that physical eye movements are taking place at the same time as the supposed internal scanning process. There is yet another potential problem with having the arrow visible: The retinal eccentricities of the different arrows may cause differences in encoding time that would sum with the supposed extrapolation times. Although Finke and Pinker (1983) demonstrated statistically that the eccentricity of an arrow and its distance to the nearest dot were not truly confounded in their Experiment 1 , again such arguments could be made stronger if we replicated the scanning effects using a paradigm in which the arrows are not presented visually.

In Experiment 3, subjects memorized a single dot pattern at the beginning of the experiment, they kept their eyes closed, and on each trial the arrow was presented auditorily as a pair of Cartesian coordinates defining the arrow's location and a clockwise angle defining its orientation. Thus, any scanning process would take place without the subjects' being able to observe the arrow or its surrounding visual field. In addition, the long delay (on the order of minutes) between the initial presentation of the pattern and the trials extends the retention interval beyond those used in Experiment 1, to a duration comparable to those employed in the scanning experiments cited in the introduction.

\section{Method}

Subjects. Sixteen Stanford undergraduates fulfilled a course requirement by participating in the experiment.

Materials. The stimulus pattern was a configuration of three dots displayed within a $25.4 \times 25.4 \mathrm{~cm}$ black border on a $28 \times 43 \mathrm{~cm}$ white card. A second card of identical size was inscribed with a black $10 \times 10$ grid, with each cell $2.54 \times 2.54 \mathrm{~cm}$; the numbers $0-10$ were inscribed on each side of the perimeter of the grid, with $(0,0)$ at the lower left corner. A third card, $20.3 \mathrm{x}$ $20.3 \mathrm{~cm}$, contained 12 numbered pips laid out in a circle corresponding to a clock face, and a $2.54-\mathrm{cm}$ arrow running through the center pointing to the 2 o'clock position.

A set of 100 arrows was created, each arrow defined by a particular grid intersection (defining its location) and a clockface number (defining the direction in which it pointed). Fifty of the arrows pointed at one of the dots in the display (or pointed to within 10 deg or less of a dot, as measured by the angle between the arrow and the line on which the origin of the arrow and the dot fell). Of these 50 arrows, 6 were for practice trials and 44 were for the experimental trials; of these 44,11 were between 2.5 and $5.1 \mathrm{~cm}$ from the nearest dot they pointed to, 11 were between 5.1 and $7.6 \mathrm{~cm}, 11$ were between 7.6 and $10.2 \mathrm{~cm}$, and 11 were between 10.2 and $12.7 \mathrm{~cm}$. Each of these sets of 11 arrows was further subdivided into three subsets, corresponding to arrows that pointed respectively to each of the three stimulus dots; two of these subsets contained 4 arrows, and the third contained 3. The sets of arrows representing the four distances were equally far, on the average, from the center of the card. Arrows pointing at $3,6,9$, or 12 o'clock occurred equally often for all four distances.

An additional set of 50 arrows was chosen such that each arrow pointed at least $30 \mathrm{deg}$ or more away from each of the dots. Six were used in the practice trials, and 44 in the experimental trials. Some of the arrows pointed away form the imag- 
inary triangle defined by the dots, and some pointed toward it; some arrows were internal to the triangle, and some external to it.

A series of 100 trials, with the first 12 being treated as practice trials in the analysis, was recorded on stereo audio tape. Each trial consisted of three numbers: a stressed clock direction followed by the unstressed word "o'clock," a stressed x-coordinate, and a stressed y-coordinate (hence, "2 o'clock, 3, 7"). The onset-to-onset time between digits was $1.2 \mathrm{sec}$. The clock direction and coordinates were recorded on the first channel, which was amplified through a speaker; the y-coordinate was also recorded on a second channel, which provided the input to a voice-operated relay interfaced to a $\mathbf{Z 8 0}$-based microcomputer. When the tape was played, the microcomputer shut the tape deck off after the $y$-coordinate had been given; when the subject pressed either of two telegraph keys, the tape deck restarted, ensuring a constant 3.8-sec intertrial interval. The RT was measured from the onset of the y-coordinate to the keypress, with a temporal grain of approximately $8 \mathrm{msec}$.

The trial sequence was chosen randomly under the constraints that there be no more than thee consecutive "yes" or "no" trials, that each block of eight consecutive trials contain an arrow at each of the four distances together with four "no" trials, and that each third of the session contain an approximately equal number of arrows for each distance pointing at each dot.

Procedure. As before, the words "image," "imagine," "imagery," "scan," and "move" were never mentioned in any of the instructions. The subjects were tested individually. They were shown the dot display, unaware of the task to follow, and were asked to memorize the locations of the dots. Memory was tested by removing the display and asking the subject to draw the dots on an identically sized blank card; the experimenter measured the positions and repeated the procedure until each dot had been drawn to within $.3 \mathrm{~cm}$ of its correct position three consecutive times (a criterion that took from three to eight attempts to meet). Next, the experimenter showed the subject the grid and explained the coordinate system to him or her, and then called out coordinate pairs and asked the subject to point as quickly as possible to the corresponding grid intersection. This was done for 10 coordinate pairs, with error feedback. The subject then had to perform this task 25 more times, but now marking the coordinate position on a blank card; each pen mark was measured with a grid printed on a transparency, and errors were pointed out. Then the clockface card was shown, and the subject was told to study the clockface and to close his or her eyes and "think about" an arrow pointing in the direction corresponding to a number between 1 and 12 called out by the experimenter. This was repeated 12 times.

The subject was then shown an arrow pointing in the direction of 3 o'clock and originating from the coordinates $(2,7)$ on the grid, and was asked to close his or her eyes, to think about new arrows defined by directions and locations recited by the experimenter, and then to open his or her eyes and point a finger to the appropriate locations and directions. Fifteen arrows were so presented, each one followed by error feedback. For 10 more trials, the subject was asked simply to think about various arrows with his or her eyes closed. The subject was then required once more to draw the dots in the original display until the dots had been reproduced to within $.64 \mathrm{~cm}$, with the dot display being shown after each attempt (in an attempt to expedite the procedure, the accuracy criterion was made less stringent in this phase).

Finally, the task was described for each subject. He or she was told that, on each trial, the tape would list a clock direction and a pair of coordinates. The task was to determine, with eyes closed, whether the arrow so defined pointed to any of the three dots in the original display. The experimenter used a drawing that showed the subject that the term "pointing to" included arrows that pointed to within $10 \mathrm{deg}$ of a dot on either side, and "not pointing to" referred to arrows pointing at least $30 \mathrm{deg}$ away from any dot. Responses were to be indicated by pressing one of two telegraph keys. The subject practiced pressing the two keys in response to the spoken commands "yes" and "no." Speed and accuracy were stressed. The tape was started and allowed to run for 12 trials; after each trial, the experimenter gave feedback about the correctness of the response. The tape then ran for 44 trials, followed by a rest period in which the subject was given the opportunity to view the dot display, followed by the remaining 44 trials.

\section{Results}

One would expect this to have been a much more difficult task for the subjects than the previous one, and indeed the subjects showed much longer RTs. Their mean error rates, $24 \%$ for misses and $17 \%$ for false alarms, were also higher. Figure 7 presents the mean latencies for correct responses to arrows that were vari. ous distances from the nearest dot, along with the corresponding error percentages, which were roughly con. stant across distance levels $(F<1)$. RTs for correct "yes" responses increased for the three greatest dis tances; however, the shortest distance yielded RT: higher than those for the two intermediate distances The variation of RT with distance was statistically significant $[F(3,45)=4.82, p<.01]$. The latency of correct "no" responses was $5,798 \mathrm{msec}$.

\section{Discussion}

The increase in RTs for the three largest dot-arrov separations are in accord with the results in the Fink and Pinker $(1982,1983)$ studies. The elevated RT fo: the shortest distance is also not unexpected, since ar elevated RT was observed for the shortest distance ir Experiment 1 and elevated error rates were observed ir Experiment 1 and in Finke and Pinker (1983). Ou explanations for these phenomena in all the studies ar closely related, and, in fact, the one we will defend her

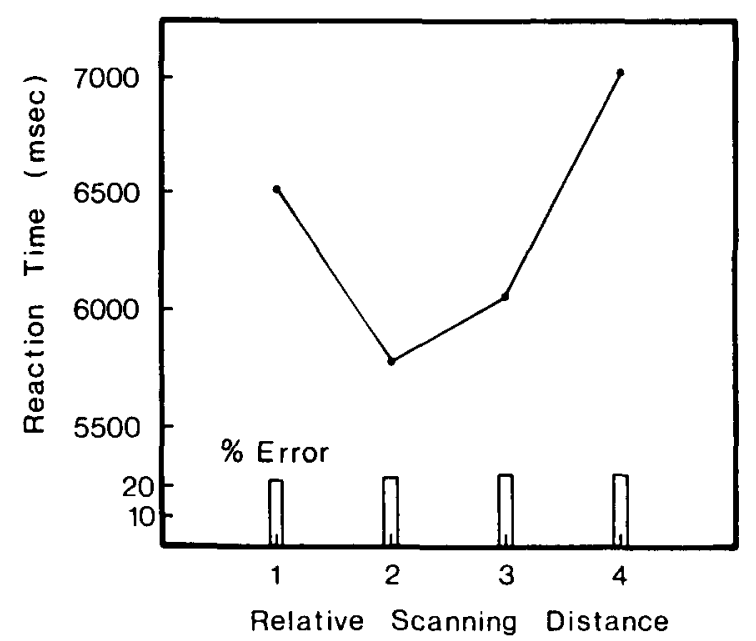

Figure 7. Mean RTs to verify that an arrow specified by it coordinates pointed at a memorized dot. Enor rates are show1 in the bar graph. 
was inspired partly by the introspections of the first author acting as a pilot subject in this experiment before Experiment 1 had been completed and partly by the introspections of several experimental subjects. It was noted that, for short distances, a moderate degree of uncertainty about the position of the dot left one in doubt as to whether to respond "yes" or "no" (see, e.g., the second and third circles in Row B of Figure 1). This often led to a time-consuming attempt to regenerate an image of the arrow from the remembered coordinates, of the dot from the memorized display, or both. Hence, trials with arrows nearest to dots took more time than one would otherwise expect; in contrast, for the three largest distances, no image regeneration was necessary for accurate responding, and the latencies reflected variation only in extrapolation times.

In fact, one can estimate the amount of uncertainty in dot position using the deviations of subjects' drawings of the dots during the training phase from the dots' true locations. The subjects were approximately .64 . $1.37 \mathrm{~cm}$ off on their first attempt, and a bit less than $.64 \mathrm{~cm}$ off on their last attempt when asked to draw the dots again at the close of the training phase. Taking $.64 \mathrm{~cm}$ as a conservative estimate and $3.8 \mathrm{~cm}$ as the midpoint of the range of distances used in the shortest distance condition, one can calculate that the subjects' image of a dot could fall approximately 9.5 deg away from an arrow extrapolated to the correct location of a dot (as measured by the angle formed by the imagined dot, the arrow location, and the extrapolated line). This is virtually identical to the maximum allowable angular deviation of a dot from an extrapolated line (10 deg) used for the "yes" trials. In comparison, a comparable positional inaccuracy combined with the second-shortest distance would yield an angular deviation of $5.7 \mathrm{deg}$, well within the allowable range for a "yes" trial. Thus, if the subjects' images of the dots were at least as inaccurate as their drawings, the images should be on the border between hits and misses for the shortest distance used and clear hits for the other distances, just what our account requires.

There are also some differences between these results and those of Finke and Pinker (1983) and Experiment 1. First, in Experiment 1, RTs were elevated only for the 0 -cm distance, not for any of the distances falling in the range of distances that produced elevated RTs here $(2.5 .5 .1 \mathrm{~cm})$. However, in Experiment 1, error rates were elevated in the $1.5-\mathrm{cm}$ distance condition, and they were also elevated in the $3 . \mathrm{cm}$ distance condition in the Finke and Pinker (1983) study. Since we already know that the increased difficulty caused by very short distances can be manifested either as elevated error rates (in Experiment $I$ and in Finke \& Pinker, 1983) or as elevated RTs (in Experiment 1), it is not necessarily an anomaly that we found increased RTs here corresponding to distances at which there had been only increased error rates in previous studies. Perhaps the great difficulty of the present task led the subjects to sacrifice speed in an attempt to keep accuracy at an acceptable level, or perhaps the longer retention interval in this task led to greater positional inaccuracy and hence a greater elevation of overall difficulty for the short distances, great enough to affect RTs at longer distances than before (recall that increased retention interval marginally interacted with arrow-dot distance in its effect on error rates in Experiment 1). In either case, there is reason to believe that the single explanation introduced in connec. tion with Figure 1 is applicable to all the extrapolation experiments.

The second discrepancy is that, in Experiment 3, scanning rates, errors, and total RTs were far higher than those in Experiment 1 and in the experiments reported by Finke and Pinker (1983). We invite the reader to attempt the task asked of our subjects in order to gain an appreciation of why this is so. Translating numerical coordinates into mentally represented locations and directions, recalling the locations of three arbitrary objects, and mentally coordinating all this information in a single image is an extremely difficult task that exacts great demands on mental resources. Since many imagery operations are chronometrically sensitive to capacity demands (Kosslyn, 1975, 1980), and since operations such as image refreshing and part generation may have to be performed many times during a single trial of an imagery task (Kosslyn, 1980), it is not surprising that both overall scanning times and the rate of scanning were slow in Experiment 3.

To summarize, the evidence from Experiment 3 points to the existence of a mental extrapolation or scanning process that can take place even when the display to be processed is retrieved from long-term memory, and even when the line to be extrapolated, the targets, and the path between them are not visible. At very short distances, additional effects of ensuring positional accuracy in the image seem to affect the overall RTs, a phenomenon that also can occur when the arrow and dot locations are visible.

\section{GENERAL DISCUSSION}

These studies replicate Finke and Pinker's (1982, 1983) finding that when people must judge whether an arrow is pointing to a dot in a previously seen pattern, they mentally scan an image of the pattern in the arrow's direction, resulting in RTs that, when the images are sufficiently accurate, increase linearly on the average with increasing distance between the arrow and the dot. Thus, the "mental scanning effect" does not depend on the subjects' receiving explicit instructions to form or scan images. The studies show, in addition, that similar functions relating RT to distance can be obtained for retention intervals ranging from 0 to $10 \mathrm{sec}$ when the pattern is seen on every trial or for retention intervals on the order of minutes when the pattern is seen only at the beginning of a block of trials. These findings suggest that the same scanning process can occur whether images 
take the form of transient sensory traces or the longer term mental reconstructions that have been the subject of previous research on mental image scanning. Furthermore, the increasing RT-distance function implicating a scanning process also can be found when subjects receive no visual input immediately before or during the scanning operation. This confirms that the effect cannot be attributed to the physical tracing of a straight line by the eyes, to the geometric properties of veridically perceived surfaces, or to the retinal eccentricity of the stimuli initiating scanning. However, when the arrow-dot distance diminishes to zero, or when the retention interval is very long and the arrow-dot distance is small, RTs increase, departing from the otherwise linear trend. Errors increase at very short distances as well, and also at longer retention intervals, both of which can be explained in terms of an interaction between displacements over time in remembered dot locations and the geometric criteria imposed by the task. Many of these findings could not be predicted by control subjects; and, indeed, it is not clear why subjects would deliberately manipulate their error rates to yield the complex results we obtained even if they were able to guess the anticipated pattern of RTs.

This series of studies strongly suggests that image scanning is not an artifact of demand characteristics or of instructions that are ambiguous between executing specific mental operations and mentally simulating physical events using tacit knowledge about the world. There is, however, another objection to the interpretation that these results implicate an image-scanning process. Pylyshyn (1981) suggested that people may mentally simulate physical events when solving geometric problems even when the task instructions do not suggest that such stimulation is called for. They simply may simulate physical events out of sheer "habit." The results in these experiments and in their predecessors cannot directly refute this particular version of the "tacit knowledge" hypothesis (although they certainly cast doubt on it, given that there is no obvious type of physical movement whose duration is a linear function of distance for all distances except very small ones and whose duration is independent of distance when the source of the journey is known beforehand). However, as Kosslyn (1981) pointed out, the "habit" version of the tacit knowledge hypothesis has no theoretical or empirical motivation whatsoever-why would people develop the habit of simulating a physical process during geometrical reasoning unless there was something in particular about their cognitive mechanisms that would make that strategy more efficient or more readily available than would more direct strategies in which physical simulation was not involved? For example, subjects could directly match the angle of the arrow against the angle of the line on which the arrow and dot lie; that angle could be found by computing a mental analog of the formula $\arctan \left[\left(y_{d}-y_{a}\right) /\left(x_{d}-x_{a}\right)\right]$, where $x_{d}, y_{d}$ are dot coordinates and $x_{a}, y_{a}$ are arrow coordinates.
Unless it is to be circular (i.e., a habit is whatever subjects do in a task), the habit hypothesis must show why a putative habit develops as the preferred strategy.

In contrast, it is possible to propose a tentative, although explicit, account of performance in this task, an account in which an image-scanning operation plays an important role. According to this account, the internal medium underlying imagery contains distinct addressable "cells" or structures representing particular locations in the visual field (Kosslyn, 1980; Trehub, 1977). The processes that operate on images and visual inputs (see Finke, 1980, and Finke \& Shepard, in press) can selectively access clusters of adjacent cells (Posner, Snyder, \& Davidson, 1980). This selective processing of a subset of the visual or imagined visual field occurs either because of capacity limitations (Posner, 1978) or because certain computational problems necessarily entail serial processing of portions of the visual field (Ullman, 1982). There is an operator that can shift this locus of attention from the cells representing one location to those representing an adjacent location a fixed small distance away in a given direction (Kosslyn, 1980; Ulman, 1982; see also Trehub, 1977, for arguments that plausible neural implementations of this shift mechanism require that it shift the attentional locus by a small constant amount). Because the attentional locus is moved in small steps, when larger shifts must be made, the process must be iterated and intermediate locations on the path must be accessed sequentially (Kosslyn, 1980; Shulman, Remington, \& McLean, 1979; Trehub, 1977; Tsal, 1983).

In addition to these image representations, patterns can be represented in a location-independent representation used in shape recognition, in which the angle formed by an object with respect to a reference frame can be represented explicitly (Marr \& Nishihara, 1978). When a person knows the frame of reference in advance, he or she can use this latter system to represent the direction of objects with respect to it. Hence, the person could decide whether or not an arrow centered on the frame of reference points to a dot by directly comparing the represented direction of the dot and the direction of the arrow, without scanning (this is what happened in Experiment 2 of Finke \& Pinker, 1983, in which subjects knew the location of the arrow in advance of its appearance). However, when subjects have no way of knowing in advance the location at which the arrow will be presented, they cannot explicitly represent the directions of the dots with respect to it. Hence, they cannot decide whether or not the arrow points to a dot simply by looking up a single vector in memory. However, subjects can instead avail themselves of the mental operation that directs the locus of attention along a linear path. They can shift that locus in the direction of the arrow until it either falls upon a dot or exits from the region of the visual field in which the dots are contained. Furthermore, the size of the attended region, which is partially under the subject's control (Posner 
et al., 1980), can be increased as the locus is moved so that the subject can keep his or her judgments consistent with the task's angular criterion for distinguishing targets from nontargets. When the region is small enough that distortions in remembered dot positions cause the dots to migrate near the edge of the attended region, the subject can allocate additional processing capacity to regenerate the image of the dot or to access its location more carefully, resulting in the increased RTs for the shortest distance found in Experiments 1 and 3 (correspondingly, if there is no such increase in capacity allocation, there will be more errors).

This account, although tentative, is fairly explicit, and its component assumptions are reasonably wellmotivated. Furthermore, it accounts for when and why mental extrapolation will take place: An extrapolation strategy will be used when people lack the information needed to represent the direction of a particular object with respect to a reference point in advance of the judgment they must make. Until a similarly explicit and motivated version of the "habit" hypothesis is outlined, one that explains why certain habits are deployed in some circumstances and not in others, it will be premature to attempt to decide between the hypotheses.

To conclude, we summarize the implications of this series of studies for the issues addressed in the introduction. By eliminating various potential artifact-based explanations of scanning phenomena and by showing that these phenomena can be generalized across several retention intervals and presentation modes, we have ensured that the capacity for image scanning and the circumstances of its use must be accounted for by any theory of imagery. In addition, we have provisionally justified its use as a technique for measuring various geometric properties in images; and we have shown that it can serve as a component of the visual routines people use to test for various geometric properties in the visual field. We stress the latter point in particular, for we suspect that mental extrapolation is a highly useful operation in any type of spatial reasoning that involves straight lines or rectilinear reference frames, such as assessing the collinearity of a set of points or line segments, judging the relative heights of two objects seen in different parts of the visual field, reading precise $y$ coordinates in a line graph, choosing the shortest unobstructed path for locomotion, or estimating distance as the crow flies.

\section{REFERENCES}

Anderson, J. R. (1978). Arguments concerning representations for mental imagery. Psychological Review, 85, 249-277.

Attreave, F., \& Pterce, C. R. (1978). Accuracy of extrapolating a pointer into perceived and imagined space. American Journal of Psychology, 91, 371-387.

BreitMeyer, B. (1983). Sensory masking, persistence, and enhancement in visual exploration and reading. In $\mathrm{K}$. Rayner (Ed.), Eye movements in reading: Perceptual and language processes. New York: Academic Press.

Finke, R. A. (1980). Levels of equivalence in imagery and perception. Psychological Review, 87, 113-132.
Finke, R. A., \& Pinker, S. (1982). Spontaneous imagery scanning in mental extrapolation. Journal of Experimental Psychology: Learning, Memory, and Cognition, 8, 142-147.

Finke, R. A., \& Pinker, S. (1983). Directional scanning of remembered visual patterns. Journal of Experimental Psychology: Learning, Memory, and Cognition, 9, 398-410.

Finke, R. A., \& Shepard, R. N. (in press). Visual functions of mental imagery. In L. Kaufman \& J. Thomas (Eds.), Handbook of perception and human performance. New York: Wiley.

Intons-Peterson, M. J. (1983). Imagery paradigms: How vulnerable are they to experimenters' expectations? Journal of Experimental Psychology: Human Perception and Performance, 9, 394-412.

KossLyn, S. M. (1973). Scanning visual images: Some structural implications. Perception \& Psychophysics, 14, 90-94.

KossLyN, S. M. (1975). Information representation in visual images. Cognitive Psychology, 7, 341-370.

KossLyN, S. M. (1978). Measuring the visual angle of the mind's eye. Cognitive Psychology, 10, 356-389.

Kosslyn, S. M. (1980). Image and mind. Cambridge, MA: Harvard University Press.

KossLyn, S. M. (1981). The medium and the message in mental imagery: A theory. Psychological Review, 83, 46-66.

Kosslyn, S. M., Ball, T. M., \& Reiser, B. J. (1978). Visual images preserve metric spatial information: Evidence from studies of imagery scanning. Journal of Experimental Psychology: Human Perception and Performance, 4, 47-60.

Kosslyn, S. M., Pinker, S., Smith, G. E., \& Shwartz, S. P. (1979). On the demystification of mental imagery. Behavioral and Brain Sciences, 2, 535-581.

Kosslyn, S. M., \& Pomerantz, J. R. (1977). Imagery, propositions, and the form of internal representations. Cognitive Psychology, 9, 52-76.

Kosglyn, S. M., Reiser, B. J., FArah, M. J., \& Fliegel, S. L. (1983). Generating visual images: Units and relations. Journal of Experimental Psychology: General, 112, 278-303.

MARR, D., \& NishihaRA, H. K. (1978). Representation and recognition of the spatial organization of three-dimensional shapes. Proceedings of the Royal Society, 200, 269-294.

McConkie, G. W., \& RAYNER, K. (1976). Identifying the span of the effective stimulus in reading: Literature review and theories of reading. In H. Singer \& R. B. Ruddoll (Eds.), Theoretical models and processes of reading. Newark, DE: International Reading Association.

Mitchell, D. B., \& Richman, C. L. (1980). Confirmed reservations: Mental travel. Journal of Experimental Psychology: Human Perception and Performance, 6, 58-66.

Phillips, W. A. (1974). On the distinction between sensory storage and short-term visual memory. Perception Psychophysics, 16, 283-290.

Pinker, S. (1980). Mental imagery and the third dimension. Journal of Experimental Psychology: General, 109, 354-371.

Pinker, S., \& Finke, R. A. (1980). Emergent two-dimensional patterns in images rotated in depth. Journal of Experimental Psychology: Human Perception and Performance, 6, 244-264.

Pinker, S., \& Kosstyn, S. M. (1978). The representation and manipulation of three-dimensional space in mental images. Journal of Mental Imagery, 1, 69-84.

Posner, M. I. (1978). Chronometric explorations of mind. Hillsdale, NJ: Erlbaum.

Posne r, M. I., Snyder, C. R., \& Davidson, B. J. (1980). Attention and the detection of signals. Journal of Experimental Psychology: General, 109, 160-174.

PotTer, M. C. (1983). Representational buffers: The eye-mind hypothesis in picture perception, reading, and visual search. In K. Rayner (Ed.), Eye movements in reading: Perceptual and language processes (pp. 413-437). New York: Academic Press.

PyLyshyn, Z. (1981). The imagery debate: Analogue media versus tacit knowledge. Psychological Review, 88, 16-45.

REED, S., Hock, H. S., \& LockHEad, G. (1983). Tacit knowledge 
and the effect of pattern configuration on mental scanning. Memory \& Cognition, 11, 137-143.

Richman, C. L., Mitchell, D. B., \& Reznik, J. S. (1979). Mental travel: Some reservations. Journal of Experimental Psychology: Human Perception and Performance, 5, 13-18.

Shulman, G. L., Remington, R. W., \& Mclean, J. P. (1979). Moving attention through space. Journal of Experimental Psychology: Human Perception and Performance, 5, 522-526.

Spoenr, K. T., \& Williams, B. E. (November 1978). Retrieving distance and location information from mental maps. Paper presented at the meeting of the Psychonomic Society, San Antonio.

ThoRNDyke, P. (1981). Distance estimation from cognitive maps. Cognitive Psychology, 13, 526-550.

Thorndyke, P., \& HAyes-Roth, B. (1982). Differences in spatial knowledge acquired from maps and navigation. Cognitive Psychology, 14, 560-589.

Trenub, A. (1977). Neuronal models for cognitive processes: Networks for learning, perception, and imagination. Journal of Theoretical Biology, 65, 141-169.

Tsal, Y. (1983). Movements of attention across the visual field. Journal of Experimental Psychology: Human Perception and Performance, 9, 523-530.

Ullman, S. (1982). Visual routines (Occasional Paper No. 20). Cambridge, MA: Massachusetts Institute of Technology, Center for Cognitive Science.

\section{NOTES}

1. The angular criteria for hits and misses also nullifies a pos- sible alternative interpretation of the linear RT-distance function in the original Finke and Pinker (1982) study: that, as distance increases, a given amount of uncertainty in the mentally represented direction of the arrow would yield increasing perpendicular distances from the dot to the extrapolated arrow, and hence greater demands on the accuracy of represented dot location. Since the criterion for hits and misses is defined in terms of angle, there is in fact no more need for positional accuracy at greater distances than at lesser ones.

2. One might suggest, however, that if subjects expect the task to be more difficult at the greater arrow-dot distances, they might then perform more cautiously at those distances, thereby increasing their RTs and reducing their error rates. But since the control subjects also predicted that the task would be more difficult at the longest delay, by this account performance should have been more accurate with increasing delay, which it was not.

3. When explaining in the questionnaire how they arrived at their answers, subjects who predicted an increasing linear relationship between distance and RT frequently reported having to undertake painstaking calculations to arrive at times proportional to the listed distances. In this regard, these control procedures are extremely conservative, since, as mentioned previously, the experimental subjects would have had neither the time nor the pencil and paper to ascertain the arrow-dot distance and calculate the appropriate RT.

(Manuscript received October 5, 1983; revision accepted for publication February 17, 1984.) 